\title{
Heart transplantation in the new era of extended donor criteria
}

\author{
Antonio Piperata ${ }^{1}$, Raphael Caraffa ${ }^{2}$, olimpia bifulco ${ }^{1}$, Martina Avesani $^{3}$, Gino Gerosa ${ }^{1}$, \\ and Tomaso Bottio ${ }^{4}$ \\ ${ }^{1}$ Affiliation not available \\ ${ }^{2}$ University of Padua \\ ${ }^{3}$ Universita degli Studi di Padova Dipartimento di Medicina \\ ${ }^{4}$ University of Padua Medical School,
}

September 25, 2021

\begin{abstract}
The heart transplantation (HT) is undoubtedly the best treatment for end-stage heart failure patients (2). However, the organ shortage remains a major challenge in cardiac surgery. Facing this problem, the medical community starts to extend the donor criteria to select more suitable organs for HT. The use of ECDs is still controversial, since it is associated with a high incidence of primary graft failure (3), and although it guarantees longer survival than without transplantation, there is still some hesitation in accepting this practice.
\end{abstract}

\section{Heart transplantation in the new era of extended donor criteria.}

Antonio Piperata ${ }^{1}$, MD; Raphael Caraffa ${ }^{2}$, MD; Olimpia Bifulco ${ }^{2}$, MD; Martina Avesani ${ }^{1}$, MD; Gino Gerosa $^{2}, \mathrm{MD}$, Tomaso Bottio ${ }^{2}, \mathrm{MD}, \mathrm{PhD}$.

${ }^{1}$ Department of Cardiology and Cardio-Vascular Surgery, Hôpital Cardiologique de Haut-Lévèque, Bordeaux University Hospital, 33604, France

${ }^{2}$ Department of Cardiac, Thoracic, Vascular Sciences and Public Health, University of Padua, via N. Giustiniani, 2, 35128 Padova, Italy

Corresponding Author: Antonio Piperata,

Avenue Magellan,33600, Pessac, FR

Phone: +33771768872

E-mail:a.piperata88@gmail.com

Keywords: Heart transplantation, marginal donors.

Word count : 448

Keyword: transplant

The authors do not have any conflict of interest to declare.

We carefully read the recent paper by N.R. Hess et al. on the use of extended criteria donors (ECDs) to increase the organ offers (1).

The heart transplantation (HT) is undoubtedly the best treatment for end-stage heart failure patients (2). However, the organ shortage remains a major challenge in cardiac surgery. 
Facing this problem, the medical community starts to extend the donor criteria to select more suitable organs for HT. The use of ECDs is still controversial, since it is associated with a high incidence of primary graft failure (3), and although it guarantees longer survival than without transplantation, there is still some hesitation in accepting this practice.

After a careful analysis of the characteristics of organ donors and the acceptance criteria used by the various centres, the authors concluded that the use of ECDs increases the number of HT without major impact on outcomes. We believe that this article gives a big boost in the debate on the use of ECDs for HT.

This is the latest demonstration that the use of ECDs in selected scenarios is safe and can be applied without increasing the mortality. This may be the results of an increased effort to improve the association between recipients and donors. In fact, it has been shown that recipient's conditions have more impact on HT outcomes than those of the donors. This strategy allows to improve the post-transplant survival at 1 and 5 years, matching high-risk organs with low-risk recipients (4).

In this context, we can also include in the ECDs group the hearts affected by coronary arteries and valvular disease, or congenital heart defects. This group of organs can successfully be transplanted with additional procedures at the time of HT. This strategy aims to optimize the performance of hearts that otherwise would not be used and that can represent the only chance for patients awaiting transplant.

We have recently published a literature review regarding the use of ECDs and concomitant procedure to increase the organ availability (5). All these experiences suggest few more considerations.

Firstly: the use of ECDs is a fundamental resource for addressing the shortage of organs.

Secondly: the profile of the recipients is constantly changing thanks to the use of mechanical circulatory supports.

Thirdly: the HT with ECDs certainly ensure better hope for patients than the absence of a transplantation.

Fourth: simple procedures including heart valve replacement or repair, coronary artery bypass grafting, or congenital heart defect repair can be successfully combined with the HT to improve the performance of these organs.

However, we strongly believe that the use of ECDs should be progressively considered by the future international recommendations and guidelines to reduce the waiting lists of patients needing HT.

\section{REFERENCES}

1. Hess NR, Seese LM, Sultan I, Wang Y, Thoma F, Kilic A. Impact of center donor acceptance patterns on utilization of extended-criteria donors and outcomes. J Card Surg. 2021 Aug 8.

2. Yancy CW, Jessup M, Bozkurt B, et al. 2017 ACC/AHA/HFSA Focused Update of the 2013 ACCF/AHA Guideline for the Management of Heart Failure: A Report of the American College of Cardiology/American Heart Association Task Force on Clinical Practice Guidelines and the Heart Failure Society of America. Circulation . 2017;136(6):e137-e161.

3. Felker GM, Milano CA, Yager JE, et al. Outcomes with an alternate list strategy for heart transplantation. J Heart Lung Transplant . 2005;24(11):1781-1786.

4. Trivedi JR, Cheng A, Ising M, Lenneman A, Birks E, Slaughter MS. Heart Transplant Survival Based on Recipient and Donor Risk Scoring: A UNOS Database Analysis. ASAIO J . 2016;62(3):297-301.

5. Piperata A, Caraffa R, Bifulco O, Avesani M, Apostolo A, Gerosa G, Bottio T. Marginal donors and organ shortness: concomitant surgical procedures during heart transplantation: a literature review. J Cardiovasc Med (Hagerstown). 2021 Aug 20. 